\title{
Constraints on primordial density perturbations from induced gravitational waves
}

\author{
Hooshyar Assadullahi and David Wands \\ Institute of Cosmology and Gravitation, University of Portsmouth, \\ Dennis Sciama Building, Burnaby Road, Portsmouth PO1 3FX, United Kingdom
}

\begin{abstract}
We consider the stochastic background of gravitational waves produced during the radiationdominated hot big bang as a constraint on the primordial density perturbation on comoving length scales much smaller than those directly probed by the cosmic microwave background or large-scale structure. We place weak upper bounds on the primordial density perturbation from current data. Future detectors such as BBO and DECIGO will place much stronger constraints on the primordial density perturbation on small scales.
\end{abstract}

\section{INTRODUCTION}

Recent cosmic microwave background (CMB) experiments [1,2] and high-redshift galaxy surveys [3] are able to probe the primordial density perturbation on cosmological scales, $\sim 10-1000 \mathrm{Mpc}$. This yields a precise measure of the primordial density contrast, $\triangle_{\mathcal{R}}^{2}\left(k_{C M B}\right)=2.5 \times 10^{-9}$ for $k_{C M B}=0.002 \mathrm{Mpc}^{-1}$ [1]. For much smaller scales, free-streaming of relativistic particles in the very early universe and Silk damping due to photon diffusion erases the primordial density perturbation on comoving scales much less than $10 \mathrm{Mpc}$ [4]. The present distribution of matter on smaller scales is the result of the subsequent non-linear evolution of the matter density and we are unable to directly relate observational data to the primordial distribution of matter. Therefore for modes which are much smaller than $10 \mathrm{Mpc}$ the primordial density perturbation remains undetermined.

One might assume that the primordial density perturbation on small scales has the same value as on larger scales. According to WMAP 5-year data $[1,2]$ the spectral index on CMB scales is smaller than one, $n_{s}=0.96_{-0.013}^{+0.014}$ ), implying that the density contrast is slightly smaller on smaller scales, but it is a huge extrapolation to assume that this is the actual scale-dependence all the way from CMB scales down to comoving scales such as $k^{-1}<10 \mathrm{pc}$, for instance, which are smaller than the comoving Hubble scale at the epoch of big bang nucleosynthesis, $T \sim \mathrm{MeV}$. The only probes of such small scales at early times are expected to be gravitational relics.

One process which allows us to put an upper bound on density perturbations is the formation of primordial black holes (PBHs) [5]. PBHs are produced when density fluctuations with a large amplitude $(\delta \rho / \rho \approx 0.01-0.1)$ enter the horizon. Their typical mass is given by the horizon mass when they were produced [6-9].

$$
M_{P B H s} \approx 10^{15}\left(\frac{t}{10^{-23} \mathrm{~s}}\right) \mathrm{g}
$$

which can be related to the temperature if they formed in the radiation dominated era

$$
M_{P B H s} \approx 10^{38}\left(\frac{\mathrm{MeV}}{T}\right)^{2} \mathrm{~g}
$$

The fact that PBHs have not been observed to date limits the initial mass fraction going into black holes, which can be used to constrain the primordial density perturbation on the corresponding scales [6]. For instance PBHs radiate Hawking radiation and thus for $M_{P B H s}<10^{15} \mathrm{~g}$ evaporations are limited because of constraints from the standard big bang nucleosynthesis (BBN), which is sensitive to the baryon-photon ratio at the time, and also because of bounds on the expected gamma-ray background. For heavier PBHs $\left(M_{P B H s}>10^{15} \mathrm{~g}\right)$, similar constraints come with the fact that the present-day density of PBHs cannot exceed the upper limit on the cold dark matter density [10].

Gravitational waves also give us a window onto density perturbations in the very early universe. They can be generated by violent events in the early universe such as bubble collisions [11], cosmic strings [12], preheating after inflation [13-16], or parametric decay of supersymmetric condensates [17, 18]. All of these mechanisms are highly model-dependent. Even gravitational waves from produced from vacuum fluctuations of the metric during inflation [19-21], though a generic prediction of inflation, have an amplitude which is dependent upon the energy scale of inflation.

In this paper we consider the bounds placed on the the primordial density perturbation from the generation of a stochastic background of gravitational waves in the very early universe. In the standard radiation-dominated hot big bang, first-order density perturbations inevitably generate gravitational waves at second (and higher) order [22-32]. Like the initial mass fraction of PBHs, the power spectrum of induced gravitational waves is determined by the primordial density perturbation and thus can place bounds on the amplitude of scalar perturbations. Indeed Saito 
and Yokoyama [34] have recently pointed out that current gravitational wave bounds are sufficient to rule out PBHs as possible candidates for intermediate mass black holes.

If we could determine the amplitude and frequency of these induced gravitational waves then we would be able to determine the primordial density perturbation when the corresponding scales crossed the Hubble scale during the early hot big bang. Throughout this paper we will use the following formula to relate the frequency of gravitational waves at the present time to the temperature at Hubble-crossing in the early radiation-dominated era [33]:

$$
\nu=\frac{c}{\lambda_{0}} \approx 1.2 \times 10^{-8} g_{*}^{1 / 6}\left(\frac{T}{\mathrm{GeV}}\right) \mathrm{Hz},
$$

where $g_{*}$ is the effective number of degrees of freedom. We are able to place upper bounds on the primordial density perturbation from BBN and cosmic microwave background (CMB) constraints and as well as current LIGO, VIRGO and pulsar timing data. If we do not detect gravitational waves with future pulsar timing $[35,36]$ and future detectors like Advanced LIGO [37], Advanced VIRGO [38], LISA [40], BBO [42] and DECIGO [43], the upper bounds on the primordial density perturbation will become significantly tighter.

We emphasize that our bounds come from adopting the standard, minimal cosmological model of adiabatic density perturbations, in their growing mode, in a radiation-dominated early universe from ultra-high energies $\left(\sim 10^{16} \mathrm{GeV}\right)$ until matter-domination when $T<\mathrm{eV}$. The quantitative constraints will be altered if one adopts non-standard cosmological evolution [44,45] such as an early matter-era (e.g., temporary domination of the energy density by massive, non-relativistic particles) or a stiff-fluid-dominated era (e.g., domination by the kinetic energy of a coherent, fast-rolling scalar field). On general grounds one expects an early era dominated by fluid "softer" than radiation, $P / \rho<1 / 3$, to dilute the fractional density of gravitational waves whose wavelength is smaller than the comoving Hubble scale (and thus behave like radiation), while the fractional density of sub-horizon gravitational waves grows relative to matter which is stiffer than radiation [47]. On the other hand the evolution of density perturbations which give rise to gravitational waves is also altered, see for example Ref. [33]. Non-adiabatic modes in a multicomponent system can lead to large-scale adiabatic density perturbations by the time of last-scattering but arise from initial isocurvature perturbations, so are not necessarily constrained by our analysis. One such example is the curvaton scenario, where the gravitational waves may be enhanced with respect to the adiabatic case if the curvaton is subdominant when it decays [48]. Such models need to be considered on a case by case basis.

This paper is organized as follows: in section 2 we introduce the basic equations used to determine the induced gravitational wave background and define the effective energy density of second-order gravitational waves. In section 3 we quantify the constraints placed on the primordial density perturbation by a variety of experiments. We present our conclusions in section 4 .

\section{SECOND-ORDER GRAVITATIONAL WAVES}

In this section we will briefly review the generation of induced gravitational waves. Details of the calculations have been described previously [30,31].

The perturbed metric in the longitudinal gauge is

$$
d s^{2}=a^{2}(\eta)\left[-(1+2 \Phi) d \eta^{2}+\left[(1-2 \Psi) \delta_{i j}+2 F_{(i, j)}+h_{i j}\right] d x^{i} d x^{j}\right]
$$

where $\Phi$ and $\Psi$ are scalar metric perturbation, $F_{i}$ is a transverse vector perturbation and $h_{i j}$ is a transverse and trace-free tensor perturbation. The scalar metric perturbations, $\Phi$ and $\Psi$, are supported by density perturbations, and in the absence of anisotropic stress we require $\Phi=\Psi$ [49]. We will find it convenient to use the Fourier transform

$$
\Phi(\mathbf{x})=\frac{1}{(2 \pi)^{\frac{3}{2}}} \int d^{3} \mathbf{k} \Phi_{\mathbf{k}} e^{i \mathbf{k} \cdot \mathbf{x}},
$$

where, for an isotropic distribution, the power spectrum is given by

$$
\left\langle\Phi_{\mathbf{k}} \Phi_{\mathbf{k}^{\prime}}\right\rangle=\frac{2 \pi^{2}}{k^{3}} \delta^{3}\left(\mathbf{k}+\mathbf{k}^{\prime}\right) \mathcal{P}(k)
$$

On large scales (much larger than the Hubble scale) the power spectrum of the primordial scalar perturbation is commonly approximated by a power law

$$
\mathcal{P}(k)=\frac{4}{9} \triangle_{\mathcal{R}}^{2}\left(\frac{k}{k_{*}}\right)^{n_{s}-1}
$$


where the numerical factor 4/9 comes from the relation between scalar metric perturbation in the longitudinal and comoving gauges on large scales in a radiation-dominated era [49].

In the second-order perturbed Einstein field equations we see the effect of first-order perturbations as a source term $\left(S_{i j}\right)$ for second-order tensor perturbations. After putting all first-order perturbation terms to the right-hand side of the Einstein field equation, we have

$$
h_{i j}^{\prime \prime}+2 \mathcal{H} h_{i j}^{\prime}+k^{2} h_{i j}=S_{i j}^{T T},
$$

where $S_{i j}^{T T}$ indicates the transverse-tracefree part of the source term. If we neglect first order tensor and vector perturbations in comparison with first order density perturbations, the right hand side of this equation is the transverse and trace-free part quadratic in first-order scalar perturbations. This behaves like a source term for induced gravitational waves $[30,31]$

$$
\begin{aligned}
S_{i j}= & 2 \Phi \partial_{i} \partial_{j} \Phi-2 \Psi \partial_{i} \partial_{j} \Phi+4 \Psi \partial_{i} \partial_{j} \Psi+\partial_{i} \Phi \partial_{j} \Phi-\partial^{i} \Phi \partial_{j} \Psi-\partial^{i} \Psi \partial_{j} \Phi+3 \partial^{i} \Psi \partial_{j} \Psi \\
& -\frac{4}{3(1+w) \mathcal{H}^{2}} \partial_{i}\left(\Psi^{\prime}+\mathcal{H} \Phi\right) \partial_{j}\left(\Psi^{\prime}+\mathcal{H} \Phi\right) \\
& -\frac{2 c_{s}^{2}}{3 w \mathcal{H}}\left[3 \mathcal{H}\left(\mathcal{H} \Phi-\Psi^{\prime}\right)+\nabla^{2} \Psi\right] \partial_{i} \partial_{j}(\Phi-\Psi)
\end{aligned}
$$

where $w=P / \rho$ is the equation of state and $c_{s}^{2}=P^{\prime} / \rho^{\prime}$ is the adiabatic sound speed.

These equations are written in the real space but in order to derive the power spectrum of gravitational waves we need to transform to Fourier space [30]

$$
h_{i j}(x, \eta)=\int \frac{d^{3} \mathbf{k}}{(2 \pi)^{\frac{3}{2}}} e^{i \mathbf{k} \cdot \mathbf{x}}\left[h_{\mathbf{k}}(\eta) e_{i j}(\mathbf{k})+\bar{h}_{\mathbf{k}} \bar{e}_{i j}(\mathbf{k})\right],
$$

where $e^{i j}(\mathbf{k})$ and $\bar{e}_{i j}(\mathbf{k})$ are the polarization tensors. The two polarization tensors $e_{i j}(k)$ and $\bar{e}_{i j}(k)$ can be given in terms of the orthonormal basis

$$
\begin{aligned}
& e_{i j}(\mathbf{k})=\frac{1}{\sqrt{2}}\left[e_{i}(\mathbf{k}) e_{j}(\mathbf{k})-\bar{e}_{i}(\mathbf{k}) \bar{e}_{j}(\mathbf{k})\right] \\
& \bar{e}_{i j}(\mathbf{k})=\frac{1}{\sqrt{2}}\left[e_{i}(\mathbf{k}) \bar{e}_{j}(\mathbf{k})+\bar{e}_{i}(\mathbf{k}) e_{j}(\mathbf{k})\right]
\end{aligned}
$$

where $\mathbf{e}$ and $\overline{\mathbf{e}}$ are unit vectors orthogonal to one another and $\mathbf{k}$ :

$$
e_{i} k^{i}=\bar{e}_{i} k^{i}=e_{i} \bar{e}^{i}=0
$$

The gravitational waves have a power spectrum in Fourier space

$$
\left\langle h_{\mathbf{k}}(\eta) h_{\mathbf{k}^{\prime}}(\eta)\right\rangle=\frac{1}{2} \frac{2 \pi^{2}}{k^{3}} \delta^{3}\left(\mathbf{k}+\mathbf{k}^{\prime}\right) \mathcal{P}_{h}(k, \eta),
$$

The effective density of a stochastic background of gravitational waves, on scales much smaller than the Hubble scale, is given by [21]

$$
\rho_{G W}=\frac{1}{32 \pi G}\left\langle\dot{h}_{i j} \dot{h}^{i j}\right\rangle=\frac{k^{2}}{32 \pi G a^{2}} \int d(\ln k) \mathcal{P}_{h}(k, \eta) .
$$

The fraction of the critical energy density in gravitational waves per logarithmic range of wavenumber $k$ in the radiation era is thus

$$
\Omega_{G W}(k, \eta)=\frac{1}{12}\left(\frac{k}{\mathcal{H}}\right)^{2} \mathcal{P}_{h}(k, \eta) .
$$

After the radiation-dominated era, the density of gravitational waves on sub-Hubble scales then redshifts exactly as any non-interacting relativistic particle species and in the present day we have

$$
\Omega_{G W, 0}(k)=\frac{\Omega_{\gamma, 0}}{12}\left(\frac{k}{\mathcal{H}}\right)^{2} \mathcal{P}_{h}(k, \eta),
$$

where we neglect additional numerical factors due to the detailed thermal history, such as the heating of photons by the annihilation of other relativistic particle species $[45,50]$. The present density of photons is $\Omega_{\gamma, 0} \simeq 4.8 \times 10^{-5}$ where here, and throughout this paper, we take $H_{0} \simeq 72 \mathrm{~km} \mathrm{~s}^{-1} \mathrm{Mpc}^{-1}$ for the present value of the Hubble rate. 


\section{CONSTRAINTS ON PRIMORDIAL DENSITY PERTURBATIONS}

In the standard cosmological scenario, second-order gravitational waves are generated during the radiationdominated era after inflation. Non-linear interactions can in principle lead to density perturbations integrated over a range of scales contributing to the gravitational wave amplitude on a given wavenumber, $k$, but in practice the second-order gravitational waves are primarily produced when first-order density perturbations on the similar on same scale, $\sim k$, come inside the Hubble scale during the radiation era [30].

Assuming a power-law spectrum for the primordial density perturbation, Eq. (7), the energy density of second-order gravitational waves, relative to the critical density at the present time, which were produced during the radiationdominated era $\left(\nu>10^{-15} \mathrm{~Hz}\right)$, can be written as

$$
\Omega_{g w, 0}(k)=F_{\text {rad }} \Omega_{\gamma, 0} \triangle_{\mathcal{R}}^{4}(k) .
$$

where, for modes which are well inside the horizon at the end of the radiation-dominated era $\left(k \eta_{e q} \gg 1\right)$, we have $[30]$

$$
F_{\text {rad }}=\frac{8}{3}\left(\frac{216^{2}}{\pi^{3}}\right) 8.3 \times 10^{-3} f_{n s}
$$

and $f_{n s}$ is weakly-dependent on the spectral tilt. $f_{n s} \approx 1$ if the density perturbation is scale-invariant [30], but becomes slightly smaller than one for a red spectrum (e.g, $f_{n s} \approx 0.97$ for $n_{s}=0.9$ ) and bigger than one for a blue spectrum (e.g, $f_{n s} \approx 1.05$ for $n_{s}=1.1$ ).

It is also possible to consider the spectrum of gravitational waves generated by density perturbations with a sharply peaked power spectrum $[30,34]$. Considering a delta-function power spectrum, $P(k)=(4 / 9) \Delta_{\mathcal{R}}^{2}\left(k_{p}\right) \delta\left(\ln \left(k / k_{p}\right)\right)$, the resulting gravitational wave spectrum is described by a sharply rising spectrum for $k<k_{p}[34]$

$$
\Omega_{g w, 0}(k)=29 \Omega_{\gamma, 0} \triangle_{\mathcal{R}}^{4}\left(k_{p}\right)\left(\frac{k}{k_{p}}\right)^{2},
$$

with an abrupt cut-off for $k>2 k_{p}$.

In the following numerical estimates we take $F_{\text {rad }} \approx 30$ in Eq. (17) corresponding to an approximately scaleinvariant spectrum of scalar perturbations, $n_{s} \approx 1$. This is expected to be a conservative lower bound on $F_{\text {rad }}$ for the blue spectra with $n_{s}>1$ required to produce a detectable background of gravitational waves on scales much smaller than the CMB scale. In the rest of this section we show, how Eq. (17) enables us to use constraints on the stochastic background of gravitational waves generated during the radiation era, $\Omega_{g w, 0}(k)$, to place upper bounds on the primordial density perturbation on the corresponding scales, $\Delta_{\mathcal{R}}^{2}(k)$. In addition Eq. (19) indicates how observational constraints on $\Omega_{g w, 0}(k)$ at a given wavenumber $k$ also places a weaker bound on the primordial density perturbation, $\Delta_{\mathcal{R}}^{2}\left(k_{p}\right) \propto\left(k_{p} / k\right)$, at higher wavenumbers, $k_{p}>k$. Our results are presented graphically in Figure .

\section{A. Cosmological density constraints}

\section{Constraint from $B B N$}

If the energy density carried by gravitational waves at the time of primordial big bang nucleosynthesis (BBN) were large, the abundances of the light nuclei produced would be altered with respect to the predictions of standard BBN. Hence, BBN can be used to constrain the total energy carried by gravitational waves at the time of nucleosynthesis $(T \simeq 1 \mathrm{MeV})[51]$

Primordial abundances of the light elements, usually quoted as a bound on the effective number of relativistic species at the time of $\mathrm{BBN}$, gives the $95 \%$ c.l. upper bound on a primordial gravitational wave background [37]

$$
\Omega_{g w, 0}<1.5 \times 10^{-5}
$$

Substituting this bound into Eq. (17) gives

$$
\triangle_{\mathcal{R}}^{2}<0.1\left(\frac{F_{\text {rad }}}{30}\right)^{-\frac{1}{2}} .
$$

This denotes the upper bound on the primordial density perturbation on the Hubble scale at the time when the gravitational waves are generated. 


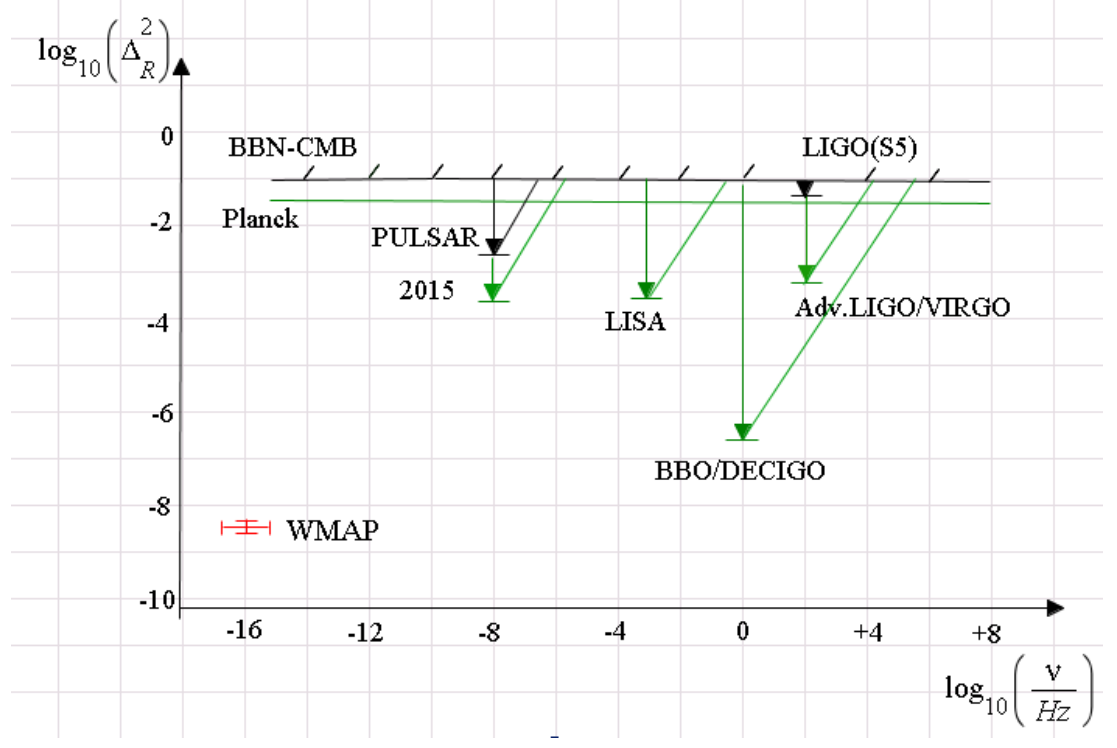

FIG. 1: Constraints on the primordial density perturbation, $\Delta_{\mathcal{R}}^{2}$, obtained from gravitational waves produced during the radiation era, using Eq. (17). Black lines denote current constraints from gravitational waves detectors and BBN. Green lines denote constraints expected from future gravitational waves detectors. Diagonal lines show the bounds on $\Delta_{\mathcal{R}}^{2}$ obtained using Eq. (19) for wavenumbers $k_{p}>k$. WMAP gives a direct measurement (shown in red) of the primordial density perturbation on very low frequencies.

Although Eq. (21) is only a weak limit on the primordial density perturbation, it is applies across a wide range of length scales. It applies on scales which are smaller than the Hubble scale at the epoch of BBN, which from Eq. (3) corresponds to frequencies $\nu>10^{-10} \mathrm{~Hz}$ today, and scales which are larger than the Hubble scale at the start of the radiation-dominated era. This is model-dependent, but in an inflationary cosmology this would be the Hubble scale at the end of reheating after inflation, which could be as large as $\nu \sim 10^{8} \mathrm{~Hz}$ for $T \sim 10^{16} \mathrm{GeV}$. If inflation occurs at lower energy scales the reheating temperature could be much lower.

The relationship between the primordial density perturbation on the CMB scale, $\triangle_{\mathcal{R}}^{2}\left(k_{C M B}\right)$, and on an arbitrary scale, $\triangle_{\mathcal{R}}^{2}(k)$, can be written as

$$
\triangle_{\mathcal{R}}^{2}(k)=\triangle_{\mathcal{R}}^{2}\left(k_{C M B}\right) \times\left(\frac{k}{k_{C M B}}\right)^{\bar{n}_{s}-1}
$$

where $\bar{n}_{s}$ describes the effective tilt between the scale $k$ and CMB scales, where $\triangle_{\mathcal{R}}^{2}$ is directly observed for $\triangle_{\mathcal{R}}^{2}\left(k_{C M B}\right)=2.5 \times 10^{-9}$ for $k_{C M B}=0.002 \mathrm{Mpc}^{-1}$ [1]. In terms of frequency we have

$$
\bar{n}_{s}=1+\frac{\log \left(\frac{\triangle_{\mathcal{R}}^{2}}{\left(\triangle_{\mathcal{R}}^{2}\left(k_{C M B}\right)\right)}\right)}{\log \left(\frac{\nu}{\nu_{C M B}}\right)},
$$

where $\nu_{C M B} \approx 10^{-18} \mathrm{~Hz}$. Therefore (21) can be interpreted as a constraint on the value of the effective spectral index, $\bar{n}_{s}$, across a wide range of scales.

According to WMAP5 data [1], the spectrum of primordial density perturbations is red ( $n_{s}$ less than unity) on CMB scales but, except for the constraints from primordial black holes (PBHs), we have no restrictions on the value of $\bar{n}_{s}$ on scales much smaller than $1 \mathrm{Mpc}$. We obtain the tightest constraint on $\bar{n}_{s}$ from a bound such as Eq. (21) applied to the smallest possible wavelength. For $\nu \simeq 10^{8} \mathrm{~Hz}$ from (21) and (23) we find

$$
\bar{n}_{s}<1.29-\frac{1}{52} \log _{10}\left(\frac{F_{r a d}}{30}\right) .
$$

Equation (24) corresponds to the maximum possible reheat temperature after inflation. However if we consider a smaller reheat temperature, for instance $T \simeq 10^{8} \mathrm{GeV}$ corresponding to $\nu \simeq 1 \mathrm{~Hz}$, we get $\bar{n}_{s}<1.44-\frac{1}{36} \log _{10}\left(\frac{F_{\text {rad }}}{30}\right)$. 


\section{Constraint from $C M B$}

A very similar bound on the effective energy density of primordial gravitational waves can be obtained around the time of last scattering of the cosmic microwave background. Again a limit on the number of massless neutrino species [52] can be translated into a bound on the gravitational wave background [53]. Unlike the BBN constraint, however, the CMB constraint depends upon the nature of inhomogeneous perturbations about the average density. For a gravitational wave background produced from a Gaussian random field of primordial density perturbations on small scales, we expect the effective energy density on long wavelengths (on scales of order $100 \mathrm{Mpc}$ ) to be independent of the density perturbations on this scale. Thus long wavelength perturbations of the gravitational wave background are non-adiabatic and Smith et al [53] give a $95 \%$ c.l. bound

$$
\Omega_{g w, 0}<1.3 \times 10^{-5},
$$

for a "homogeneous" gravitational wave background. This is marginally stronger than the BBN constraint (20). It gives effectively the same constraint on the primordial density perturbation (21), and the effective spectral index (24), but extends to longer wavelengths $\sim 10^{-15} \mathrm{~Hz}$, corresponding to scales inside the Hubble scale at the time of last-scattering.

Future data from CMB experiments such as Planck and the proposed CMBPol mission are expected to improve the CMB bound. For Planck the expected bound corresponds to $\Omega_{g w, 0}<2.7 \times 10^{-6}[53]$ which would bound

$$
\triangle_{\mathcal{R}}^{2}<0.04\left(\frac{F_{\text {rad }}}{30}\right)^{-\frac{1}{2}} .
$$

\section{B. Constraints from ground-based detectors}

\section{Current LIGO/VIRGO}

We can obtain a tighter constraint on the primordial density perturbation on scales probed by direct detectors, such as the Laser Interferometer Gravitational Wave Observatory (LIGO) [37] and gravitational wave detector at the European gravitational observatory (VIRGO) [38]. LIGO's maximum sensitivity is around a frequency, $\nu=100 \mathrm{~Hz}$. The latest results from the LIGO S5 science run give a bound on the energy density of gravitational waves on this scale [39]

$$
\Omega_{g w, 0}<6.9 \times 10^{-6} .
$$

Hence from Eq. (17) the constraint on the density perturbation on the LIGO/VIRGO scale is

$$
\triangle_{\mathcal{R}}^{2}<0.07\left(\frac{F_{r a d}}{30}\right)^{-\frac{1}{2}} .
$$

This is a slightly tighter bound than that currently obtained from BBN and the CMB, Eq. (21), however unlike the $\mathrm{BBN}$ and CMB bound it only applies to LIGO/VIRGO scales. The corresponding constraint on $\bar{n}_{s}$ on this scale comes from Eq. (23)

$$
\bar{n}_{s}<1.37-\frac{1}{40} \log _{10}\left(\frac{F_{r a d}}{30}\right) .
$$

\section{Advanced LIGO/VIRGO}

Advanced LIGO/VIRGO will give us an improved constraint on a stochastic background of gravitational waves on the same scales $[38,54]$. The smallest density of gravitational waves which could be detected by Advanced LIGO/VIRGO is $10^{3}$ times smaller than current LIGO/VIRGO bounds. Considering the smallest detectable energy density $\Omega_{g w, 0}<10^{-9}$ in Eq. (17) returns

$$
\triangle_{\mathcal{R}}^{2}<8 \times 10^{-4}\left(\frac{F_{r a d}}{30}\right)^{-\frac{1}{2}}
$$


Equation (23) gives the expected constraint on $\bar{n}_{s}$ from Advanced LIGO/VIRGO (taking $\nu=100 \mathrm{~Hz}$ )

$$
\bar{n}_{s}<1.27-\frac{1}{40} \log _{10}\left(\frac{F_{r a d}}{30}\right) .
$$

\section{Constraints from LISA}

The Laser Interferometer Space Antenna (LISA) is the first gravitational wave detector planned in space and is the most sensitive detector currently planned. Assuming LISA's instrumental noise is well-behaved [55], it could detect a stochastic background of gravitational waves at a level $\Omega_{g w, 0} \sim 10^{-11}$ at frequencies $\nu_{L I S A} \sim 10^{-3} \mathrm{~Hz}$ $[40,41]$. However the sensitivity of LISA leads to many potential overlapping sources and hence the problem of source confusion. In particular the astrophysical background from unresolved extra-galactic white-dwarf binaries is expected to limit LISA's ability to distinguish a primordial gravitational wave background to $[55,56]$

$$
\Omega_{g w, 0}<10^{-10} .
$$

The corresponding upper bound on the primordial density perturbation on LISA scales comes from (17):

$$
\triangle_{\mathcal{R}}^{2}<3 \times 10^{-4}\left(\frac{F_{\text {rad }}}{30}\right)^{-\frac{1}{2}}
$$

The constraint on $\bar{n}_{s}$ on LISA scales comes from Eq. (23)

$$
\bar{n}_{s}<1.34-\frac{1}{30} \log _{10}\left(\frac{F_{r a d}}{30}\right) .
$$

This is a slightly weaker bound on the effective spectral index compared with Advanced LIGO, as LISA is sensitive on length scales much larger than LIGO scales.

\section{Constraints from BBO/DECIGO}

The Big Bang Observer (BBO) [42] and the DECi-hertz Interferometer Gravitational wave Observatory (DECIGO) [43] are ambitious proposals for future space-based observatories to detect cosmological gravitational waves. They should be able to detect a stochastic background of gravitational waves down to an effective energy density $\Omega_{g w, 0} \approx$ $10^{-16}$ at $\nu_{B B O} \approx 1 \mathrm{~Hz}$. This waveband is chosen to avoid the confusion noise due to white dwarf binary mergers which cuts off above $0.2 \mathrm{~Hz}$. The designs of BBO and DECIGO are based on the requirement to identify and remove the remaining foregrounds from neutron star and black hole binaries [57].

If induced gravitational waves during the radiation era are not detected with BBO/DECIGO, then we will be able to place a tight constraint on the primordial density perturbation and hence $\bar{n}_{s}$ on this scale $(1 \mathrm{~Hz})$. From (17) and (23) we obtain

$$
\triangle_{\mathcal{R}}^{2}<3 \times 10^{-7}\left(\frac{F_{r a d}}{30}\right)^{-\frac{1}{2}}
$$

and hence

$$
\bar{n}_{s}<1.11-\frac{1}{36} \log _{10}\left(\frac{F_{r a d}}{30}\right) .
$$

\section{E. Constraints from pulsar timings}

Analysis of pulse data from pulsars shows that they are very stable clocks. Measurement of timing residuals, which is the difference between the observed time of arrival and predicted time of arrival, can in principle be used to directly detect gravitational waves passing between the pulsar and the observer $[35,36]$. The data from current 
observations of an array of pulsars places an upper bound on the stochastic background of gravitational waves, with periods comparable to the total observation time span. This is typically $1-10$ years, and corresponds to $10^{-8}-10^{-9}$ Hz. For $\nu=10^{-8} \mathrm{~Hz}$, the constraint on the present density of gravitational waves is [36]

$$
\Omega_{g w, 0}<4 \times 10^{-8} \text {. }
$$

Substituting (37) in (17), gives us the current constraints on the primordial density perturbation

$$
\triangle_{\mathcal{R}}^{2}<5 \times 10^{-3}\left(\frac{F_{\text {rad }}}{30}\right)^{-\frac{1}{2}} .
$$

The constraint on $\bar{n}_{s}$ comes from (17)

$$
\bar{n}_{s}<1.63-\frac{1}{20} \log _{10}\left(\frac{F_{\text {rad }}}{30}\right) .
$$

Saito and Yokoyama [34] have recently used similar constraints, on the induced gravitational wave background from pulsar timing arrays, to rule out the large amplitude of primordial density perturbations required to produce any significant number of primordial black holes in the intermediate mass range, $4 \times 10^{2} M_{\odot} \leq M_{P B H} \leq 5 \times 10^{3} M_{\odot}$, corresponding to $8 \times 10^{35} \mathrm{~g} \leq M_{P B H} \leq 10^{37} \mathrm{~g}$, which from Eq. (2) would have formed at temperatures $T \approx 3-10 \mathrm{MeV}$.

Future pulsar timing will give a better constraint. If gravitational waves are not detected, the upper limit, based on timing 20 pulsars over 5 years, would be $\Omega_{g w, 0}<10^{-10}$ [36]. From (17), the future constraint on the primordial density perturbation in five years time for $\nu=10^{-8} \mathrm{~Hz}$ would be

$$
\triangle_{\mathcal{R}}^{2}<3 \times 10^{-4}\left(\frac{F_{\text {rad }}}{30}\right)^{-\frac{1}{2}} .
$$

The future constraint on $\bar{n}_{s}$ would then be

$$
\bar{n}_{s}<1.50-\frac{1}{20} \log _{10}\left(\frac{F_{r a d}}{30}\right)
$$

\section{CONCLUSION}

Despite remarkable recent progress in astronomical observations mapping density perturbations on large scales (10$1000 \mathrm{Mpc}$ ) in our Universe, we know little about the primordial distribution of matter on much smaller scales. This is due to Silk damping and free-streaming of relativistic particles in the early universe, and subsequent non-linear evolution of matter perturbations at much later times. The only constraints on these scales come from gravitational relics of the very early universe. Previous work has focussed on the possible formation of primordial black holes from large over-densities.

In this paper we have shown how limits on a stochastic background of gravitational waves can be used to place limits on density perturbations in the early radiation-dominated era of the standard hot big bang cosmology.

BBN and CMB limits on a primordial gravitational wave background places only a weak constraint on the amplitude of primordial density perturbations, $\triangle_{\mathcal{R}}^{2}<0.1$, but this applies across a wide range of frequencies, from $10^{-15} \mathrm{~Hz}$ to frequencies as high as $10^{8} \mathrm{~Hz}$, depending on the maximum temperature at the start of the radiation-dominated era. By contrast, gravitational wave detectors such as LIGO and VIRGO place slightly tighter bounds, currently $\triangle_{\mathcal{R}}^{2}<0.07$, but only over a narrower range determined by the frequency response of the detector.

Future gravitational wave experiments offer the prospect of much tighter bounds on, or a detection of, a stochastic gravitational wave background and hence the primordial density perturbation on small scales. A space-based experiment such as LISA could detect gravitational waves produced by density perturbations $\triangle_{\mathcal{R}}^{2} \sim 10^{-4}$, and future data from pulsar timing arrays could have similar sensitivity. The most ambitious current proposed gravitational wave observatories including BBO and DECIGO offer the prospect of detecting gravitational waves as small as $\triangle_{\mathcal{R}}^{2} \sim 10^{-7}$.

If gravitational wave background is not detected by these experiments it would imply that the primordial power spectrum remains close to scale-invariant, or decreases in power on small scales, $\bar{n}_{s}<1.29$, which provides a valuable new constraint on models for the origin of structure. Nonetheless it remains a challenge to design an experiment that could detect gravitational waves produced by primordial density perturbations of the same power, $\triangle_{\mathcal{R}}^{2} \sim 10^{-9}$, as seen on the largest scales in the universe today. 
We have assumed the simplest expansion history of the universe, being radiation dominated from very early times, corresponding to temperatures as high as $10^{16} \mathrm{GeV}$. If the early universe has a more complicated history, the constraints may be altered. A period of inflation is expected to dilute pre-existing gravitational waves on sub-Hubble scales, without generating a significant additional background [58]. On the other hand although an early matterdominated era before BBN, such as the reheating or preheating after inflation, would also dilute any gravitational waves that had already been generated, it could itself produce significant tensor metric perturbations on scales that re-enter the Hubble scale during an early matter-dominated era [33]. We leave a more detailed investigation of constraints on the primordial density perturbation in more general cosmological scenarios to future work.

\section{Acknowledgements}

We are grateful to Marco Bruni, Karen Masters and Misao Sasaki for useful comments. HA is supported by the British Council (ORSAS). DW is supported by the STFC.

[1] E. Komatsu et al. [WMAP Collaboration], Astrophys. J. Suppl. 180, 330 (2009) [arXiv:0803.0547 [astro-ph]].

[2] G. Hinshaw et al. [WMAP Collaboration], Astrophys. J. Suppl. 180, 225 (2009) [arXiv:0803.0732[astro-ph]].

[3] B. A. Reid et al., arXiv:0907.1659 [astro-ph.CO].

[4] J. A. Peacock, Cambridge, UK: Univ. Pr. 682 p (1999).

[5] B. J. Carr and S. W. Hawking, Mon. Not. Roy. Astron. Soc. 168, 399 (1974).

[6] A. M. Green and A. R. Liddle, Phys. Rev. D 56, 6166 (1997) [arXiv:astro-ph/9704251].

[7] D. H. Lyth, K. A. Malik, M. Sasaki and I. Zaballa, JCAP 0601, 011 (2006) [arXiv:astro-ph/0510647].

[8] B. J. Carr, [arXiv:astro-ph/0504034].

[9] K. Kohri, D. H. Lyth and A. Melchiorri, JCAP 0804, 038 (2008) [arXiv:0711.5006 [hep-ph]].

[10] I. Zaballa, A. M. Green, K. A. Malik and M. Sasaki, JCAP 0703, 010 (2007) [arXiv:astro-ph/0612379].

[11] M. Kamionkowski, A. Kosowsky and M. S. Turner, Phys. Rev. D 49, 2837 (1994) [arXiv:astro-ph/9310044].

[12] B. Allen and E. P. S. Shellard, Phys. Rev. D 45, 1898 (1992).

[13] S. Y. Khlebnikov and I. I. Tkachev, Phys. Rev. D 56, 653 (1997) [arXiv:hep-ph/9701423].

[14] R. Easther, J. T. . Giblin and E. A. Lim, Phys. Rev. Lett. 99, 221301 (2007) [arXiv:astro-ph/0612294].

[15] R. Easther, J. T. Giblin and E. A. Lim, Phys. Rev. D 77, 103519 (2008) [arXiv:0712.2991 [astro-ph]].

[16] J. F. Dufaux, G. N. Felder, L. Kofman and O. Navros, JCAP 0903, 001 (2009) [arXiv:0812.2917 [astro-ph]].

[17] J. F. Dufaux, Phys. Rev. Lett. 103, 041301 (2009) [arXiv:0902.2574 [astro-ph.CO]].

[18] A. Kusenko, A. Mazumdar and T. Multamaki, Phys. Rev. D 79, 124034 (2009) [arXiv:0902.2197 [astro-ph.CO]].

[19] A. A. Starobinsky, JETP Lett. 30, 682 (1979) [Pisma Zh. Eksp. Teor. Fiz. 30, 719 (1979)].

[20] M. S. Turner, Phys. Rev. D 55, 435 (1997) [arXiv:astro-ph/9607066].

[21] M. Maggiore, Phys. Rept. 331, 283 (2000) [arXiv:gr-qc/9909001].

[22] K. Tomita, Prog. Theor. Phys. 37, 831 (1967).

[23] S. Matarrese, O. Pantano and D. Saez, Phys. Rev. Lett. 72, 320 (1994) [arXiv:astro-ph/9310036].

[24] S. Matarrese and S. Mollerach, arXiv:astro-ph/9705168.

[25] S. Matarrese, S. Mollerach and M. Bruni, Phys. Rev. D 58, 043504 (1998) [arXiv:astro-ph/9707278].

[26] H. Noh and J. c. Hwang, Phys. Rev. D 69, 104011 (2004).

[27] C. Carbone and S. Matarrese, Phys. Rev. D 71, 043508 (2005) [arXiv:astro-ph/0407611].

[28] K. Nakamura, Prog. Theor. Phys. 117, 17 (2007) [arXiv:gr-qc/0605108]; Phys. Rev. D 74, 101301 (2006) [arXiv:grqc/0605107].

[29] K. A. Malik and D. Wands, Phys. Rept. 475, 1 (2009) [arXiv:0809.4944 [astro-ph]].

[30] K. N. Ananda, C. Clarkson and D. Wands, Phys. Rev. D 75, 123518 (2007) [arXiv:gr-qc/0612013].

[31] D. Baumann, P. J. Steinhardt, K. Takahashi and K. Ichiki, Phys. Rev. D 76, 084019 (2007) [arXiv:hep-th/0703290].

[32] A. Mangilli, N. Bartolo, S. Matarrese and A. Riotto, Phys. Rev. D 78, 083517 (2008) [arXiv:0805.3234 [astro-ph]].

[33] H. Assadullahi and D. Wands, Phys. Rev. D 79083511 (2009) arXiv:0901.0989 [astro-ph.CO].

[34] R. Saito and J. Yokoyama, Phys. Rev. Lett. 102, 161101 (2009) [arXiv:0812.4339 [astro-ph]].

[35] F. A. Jenet, G. B. Hobbs, K. J. Lee and R. N. Manchester, Astrophys. J. 625, L123 (2005) [arXiv:astro-ph/0504458].

[36] F. A. Jenet et al., Astrophys. J. 653, 1571 (2006) [arXiv:astro-ph/0609013].

[37] B. Abbott et al. [LIGO Collaboration], Astrophys. J. 659, 918 (2007) [arXiv:astro-ph/0608606].

[38] F. Acernese et al., Class. Quant. Grav. 25, 184001 (2008).

[39] B. Abbott et al. [LIGO Collaboration], Nature 460, 990 (2009).

[40] S. A. Hughes, arXiv:0711.0188 [gr-qc].

[41] B. S. Sathyaprakash and B. F. Schutz, Living Rev. Rel. 12, 2 (2009) [arXiv:0903.0338 [gr-qc]].

[42] V. Corbin and N. J. Cornish, Class. Quant. Grav. 23, 2435 (2006) [arXiv:gr-qc/0512039].

[43] S. Kawamura et al., J. Phys. Conf. Ser. 120, 032004 (2008). 
[44] N. Seto and J. Yokoyama, J. Phys. Soc. Jap. 72, 3082 (2003) [arXiv:gr-qc/0305096].

[45] L. A. Boyle and P. J. Steinhardt, Phys. Rev. D 77, 063504 (2008) [arXiv:astro-ph/0512014].

[46] M. S. Turner, Phys. Rev. D 28, 1243 (1983).

[47] M. Giovannini, Phys. Lett. B 668, 44 (2008) [arXiv:0807.1914 [astro-ph]].

[48] N. Bartolo, S. Matarrese, A. Riotto and A. Vaihkonen, Phys. Rev. D 76, 061302 (2007) [arXiv:0705.4240 [astro-ph]].

[49] V. F. Mukhanov, H. A. Feldman and R. H. Brandenberger, Phys. Rept. 215, 203 (1992).

[50] K. Nakayama, S. Saito, Y. Suwa and J. Yokoyama, JCAP 0806, 020 (2008) [arXiv:0804.1827 [astro-ph]].

[51] R. H. Cyburt, B. D. Fields, K. A. Olive and E. Skillman, Astropart. Phys. 23, 313 (2005) [arXiv:astro-ph/0408033].

[52] E. Pierpaoli, Mon. Not. Roy. Astron. Soc. 342, L63 (2003) [arXiv:astro-ph/0302465].

[53] T. L. Smith, E. Pierpaoli and M. Kamionkowski, Phys. Rev. Lett. 97, 021301 (2006) [arXiv:astro-ph/0603144].

[54] D. Sigg [LIGO Scientific Collaboration], Class. Quant. Grav. 25, 114041 (2008).

[55] C. J. Hogan and P. L. Bender, Phys. Rev. D 64, 062002 (2001) [arXiv:astro-ph/0104266].

[56] N. J. Cornish, Phys. Rev. D 65, 022004 (2002) [arXiv:gr-qc/0106058].

[57] C. Cutler and J. Harms, Phys. Rev. D 73, 042001 (2006) [arXiv:gr-qc/0511092].

[58] F. Arroja, H. Assadullahi, K. Koyama and D. Wands, to appear in Physical Review D, arXiv:0907.3618 [astro-ph.CO]. 